\title{
ESTUDO DA ÚLCERA DE PRESSÃO POR MEIO DE TERMOGRAFIA EM PACIENTE ACAMADO COM ESCLEROSE MÚLTIPLA
}

\author{
Gladis Aparecida Galindo Reisemberger de Souza', Maria Lucia Leite Ribeiro Okimoto², Ramón \\ Sigifredo Cortés Paredes ${ }^{3}$, Maria Cristina Pacheco ${ }^{4}$, Luis Henrique Stocco da Silva ${ }^{5}$, Carlos Dalmaso \\ Neto ${ }^{6}$
}

1 Doutoranda, PGMEC, Universidade Federal do Paraná (UFPR)

2 Professora Doutora, PGMEC Universidade Federal do Paraná (UFPR)

3 Professor Doutor, PGMEC Universidade Federal do Paraná (UFPR)

${ }^{4}$ Mestranda, PGMEC Universidade Federal do Paraná (UFPR)

5 Doutorando (UFPR), Professor Universidade Positivo, Curitiba, PR

${ }^{6}$ Médico. Pós-graduado em Termologia e Termografia, FMUSP

Resumo: 0 presente trabalho, ocupa-se de uma análise por Termografia, para verificação dos pontos de pressão de paciente que se encontra acamado e praticamente sem movimentos, tais pontos de contato, futuramente, podem transformar-se em escaras. O objetivo deste estudo é a observação de pontos quentes (hotspots) em paciente com esclerose múltipla, por meio de imagens termográficas (termogramas), que, com as análises medindo calor, relatam a preexistência de inflamação. Pela investigação precoce do paciente acamado é possível identificar, evitando em tempo, o aparecimento de úlceras de pressão, também chamadas de escaras. Como metodologia foi realizada uma análise para verificação de pontos de pressão por Termografia e um questionamento junto ao paciente e seu cuidador, sobre seus pontos de maior incômodo. Para aquisição das imagens infravermelhas, foi utilizada uma câmera com Sensor ThermaCAM T400 (FLIR Systems Inc., North Billerica, EUA). Os resultados do estudo Termográfico, na correlação entre temperatura cutânea e pontos de pressão, demonstram a sensibilidade da área afetada, sendo possível intervir para que posteriormente o paciente não sofra com as escaras, o que é bastante complicado para o indivíduo. É possível verificar que nos locais do corpo onde o paciente reclama de dores intensas, a demonstração da sensibilidade, que é aferida nas imagens por infravermelho, revela que tais pontos devem ser observados com cautela.

Palavras-chave: Úlcera de Pressão; Termografia; Esclerose Múltipla

\begin{abstract}
The present work deals with an analysis by Thermography, to check the pressure points of patients who are bedridden and practically without movements, such points of contact, in the future, can become eschar. The objective of this study is to observe hot spots (hotspots) in a patient with multiple sclerosis, through thermograms, which, with the analyzes measuring heat, report the preexistence of inflammation. By early investigation of the bedridden patient it is possible to identify, avoiding in time, the appearance of pressure ulcers, also called bedsores. As methodology, an analysis was carried out to verify pressure points by Thermography and a questioning of the patient and his caregiver on the most uncomfortable points. For acquisition of infrared images, a camera with ThermaCAM T400 Sensor (FLIR Systems Inc, North Billerica, USA) was used. The results of the Thermographic study, in the correlation between cutaneous temperature and pressure points, demonstrate the sensitivity of the affected area, and it is possible to intervene so that the patient does not subsequently suffer from the bedsores, which is quite complicated for the individual. It is possible to verify that in the places of the body where the patient complains of intense pain, the demonstration of the sensitivity, which is measured in the infrared images, reveals that such points must be observed with caution.
\end{abstract}

Keywords: Pressure Ulcer; Thermography; Multiple Sclerosis 


\section{INTRODUÇÃO}

Dentro do contexto atual, sabe-se que o doente, quando acamado por longo período, está sujeito a sofrer com feridas, por se encontrar estático e somente em contato com a cama, cadeira, etc ${ }^{1}$. Para promover conforto aos pacientes é necessário a tomada de alguns cuidados, muitas vezes não observados a tempo. Diante disto, há a necessidade de identificar precocemente as feridas nestes pacientes, para prevenção.

Feridas, ulceras' de pressão, intituladas também de ulceras de compressão ou ulceras de decúbito, são conceituadas feridas crônicas que acometem áreas onde encontra-se pressão sobre proeminências ósseas, ísquio, sacro, calcâneo, trocânter, região occipital, dorso do pé, maléolo e a patela. Estas podem se desenvolver de 24 horas até cinco dias, antes de se manifes$\operatorname{tar}^{1}$. Pessoas com deficiência motora, que passam a maior parte do tempo de suas vidas acamadas, na dependência de um cuidador, um requisito básico necessário é o cuidado com sua movimentação e conforto ${ }^{1}$ além disso, uma inspeção meticulosa diária da pele do indivíduo restrito ao leito, podendo-se, desta forma, detectar precocemente a hiperemia, ocupando-se de uma ação imediata, para evitar que ocorra a ruptura da pele, no entanto, a dificuldade em obter a resposta sobre possíveis feridas é evidente.

A Esclerose múltipla (EM) é uma doença crônica neuroinflamatória, autoimune, que leva à disfunção neurológica progressiva, comprometendo Sistema Nervoso Central (SNC). ${ }^{2}$ A doença destrói a mielina, proteína fundamental na transmissão do impulso nervoso. Embora as características clínicas sejam bem conhecidas, os aspectos etiológicos ainda estão em estudo. Com o aprofundamento dos conhecimentos imunopatológicos, têm-se ampliado as perspectivas terapêuticas. ${ }^{3}$
EM é uma doença que está progredindo em número de casos no mundo. No Brasil estudos realizados em São Paulo, mostram taxa de prevalência em adultos jovens de 20 a 40 anos de aproximadamente 5/100 000 habitantes (cinco casos por 100 mil habitantes). Sendo ela mais comum nas mulheres (2 para 1).4, 5, 6

A Termografia é uma técnica que capta a radiação infravermelha emitida pelos corpos, possibilitando a medição de temperaturas e a observação de padrões de distribuição de calor em um determinado sistema. É um exame inócuo, sem emissão de radiação ionizante, indolor, de baixo custo, rápido na captura de imagens e de ampla aplicação. A avaliação é feita basicamente pela simetria do corpo humano e pela termorregulação. Existe uma vasta gama de aplicações da termografia em diversas áreas, tais como: militar, medicina, indústria e engenharia. ${ }^{7,8}$

Por intermédio de interpretação e captura de gradientes térmicos, entre as várias partes do corpo, é possível detectar anormalidades na sua fase mais precoce, prevenindo lesões. Adicionalmente, a metodologia pode ser usada para acompanhamento da evolução da doença ao longo do tempo e pós-tratamentos ${ }^{9}$.

Monitoramento de úlcera de pressão ajuda profissionais no foco da redução da incidência e na gravidade das feridas. 0 monitoramento em pontos críticos como proeminências ósseas podem ser muito úteis. Desta forma há a necessidade de tecnologias que comprovem a existência de pontos propícios que se transformarão em feridas. O objetivo do trabalho foi investigar a possibilidade de prevenir ulceras de pressão por meio de inspeções da pele por Termografia. 


\section{LPAJMT METODOLOGIA}

A fim de verificar as necessidades e o desconforto do voluntário acometido por esclerose múltipla de grau avançado, que aceitou participar do estudo, foi utilizado uma análise para verificação de pontos de pressão por Termografia e um questionamento junto ao paciente e seu cuidador, sobre seus pontos de maior incômodo. 0 paciente assinou termo de consentimento livre e esclarecido, atendendo a resolução 466/2012-CNS-MS, para o estudo.

Para aquisição das imagens infravermelhas, foi utilizada uma câmera com Sensor ThermaCAM T400 (FLIR Systems Inc., North Billerica, EUA). A câmera possui um detector do tipo uncooled focal plane (FPA), i.e., microbolômetro sem refrigeração, de quarta geração, com 320x240 pixels (76.800 pixels), que atua na faixa espectral de ondas eletromagnéticas entre 7,5 a $13 \mu \mathrm{m}$, o que corresponde à faixa do infravermelho longo (FIR) para estudo na faixa de temperatura $-20^{\circ} \mathrm{C}$ até $+350^{\circ} \mathrm{C}$. $\mathrm{O}$ equipamento permite obter imagens com resolução espacial (IFOV) de 1,4mrad, para visualização de pontos quentes de 1,4mm a distâncias de 1 metro, com lente padrão e sem lentes adicionais. A precisão de temperatura reportada pelo fabricante é de $\pm 1^{\circ} \mathrm{C}$ e resolução de temperatura de $0,05^{\circ} \mathrm{C}$ a $30^{\circ} \mathrm{C}$.

Um dos métodos realizados, foi um levantamento para averiguação de possíveis pontos de desconforto no paciente. Para tal, efetuou-se como procedimento técnico, uma investigação fazendo primeiramente perguntas ao voluntário sobre seus pontos de desconforto, depois foi desenhado um boneco em forma humana para marcação desses pontos e classifica-los com números, medindo seu grau de desconforto, e com isso poder também realizar estudos em pessoas que passam a maior parte do tempo em cadeira de rodas e ou deitadas, como pacientes com EM por exemplo.

Outro fator levantado foi o resultado do exame de termografia realizado com o paciente, que apontou vários pontos de alteração de temperatura. Tais pontos poderão levar ao desenvolvimento de úlceras de pressão em função de sua limitação de movimentos na cama.

Foi realizado exame por Termografia para verificação de pontos de calor, para monitoramento do indivíduo, na tentativa de prevenir escaras. Por apresentar esclerose múltipla avançada, com comprometimento da musculatura dos membros inferiores e parte dos superiores, verificou-se muitos pontos com assimetria térmica, quando analisado por Termografia, em função da fisiologia do paciente não estar dentro dos padrões de normalidade.

O exame cumpriu-se na própria residência do paciente e foi utilizada a câmera termográfica FLIR T400 a fim de capturar imagens térmicas de regiões de seu corpo, para averiguação de hotspots, que futuramente podem transformar-se em escaras. As imagens foram feitas por um médico especialista na área de Termografia e por uma profissional na área de Tecnologia Radiológica, também certificada em Termografia. Durante toda a realização do exame o paciente foi sendo posicionado pelos examinadores e por seu cuidador.

Após captação, as imagens foram colocadas em um computador e seus termogramas analisados por meio de um software específico de análise de imagens térmicas (FLIR Tools ${ }^{\circledR}$ ). 


\section{RESULTADOS}

Os resultados do estudo Termográfico, na correlação entre temperatura cutânea e pontos de pressão, demonstram a sensibilidade da área afetada, sendo possível intervir para que posteriormente o paciente não sofra com as escaras, o que é bastante complicado para o indivíduo.

Os pontos de desconforto em seu corpo, são exibidos na figura $\mathbf{1} \mathrm{com}$ marcas coloridas, em regiões incômodas relatadas pelo paciente, bem como o grau (em números) deste desconforto.
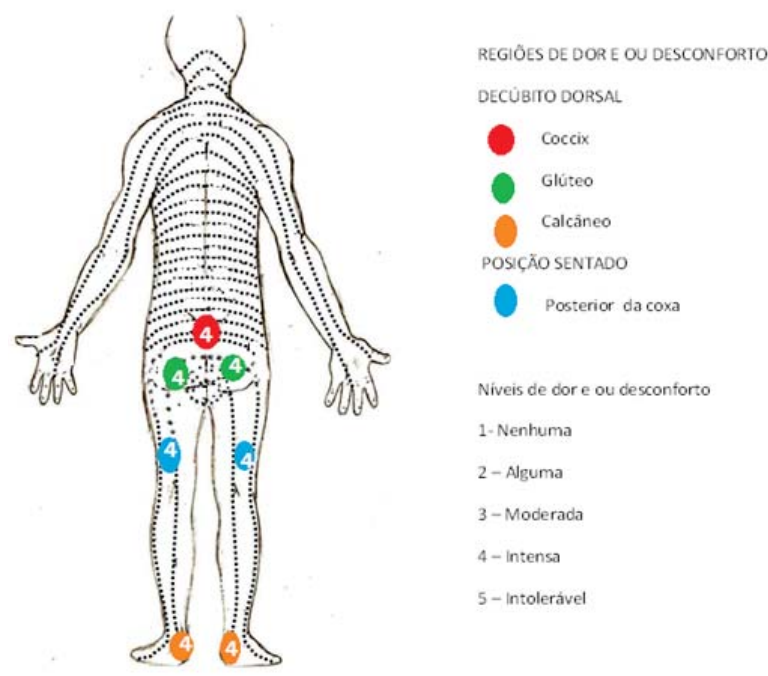

Niveis de dor e ou desconforto

1- Nenhuma

2-Alguma

3-Moderada

$4-$ intensa

5 -Intolerável

FIGURA 1 Desenho da figura humana demonstrando locais de desconforto, quando em posiçẫo ortostática e sentada.

É possível verificar que nos locais do corpo onde o paciente reclama de dores intensas (nível 4), documentado pelo desenho da figura 1, a demonstração da sensibilidade, que é aferida nas imagens por infravermelho, evidencia os pontos a serem observados com cautela, já que há a diferença $(\triangle)$ de no mínimo $1,5^{\circ} \mathrm{C}$ entre as regiões com lesão e partes vizinhas saudáveis, e segundo Judy, é determinado que uma variação de temperatura de $1,5^{\circ} \mathrm{C}$ é capaz de prever com precisão o desenvolvimento de ulceras de pressão. ${ }^{10}$

$\mathrm{Na}$ figura 2 pode-se analisar os pontos relacionados às partes do corpo com objetivo de melhor compreensão de localização de pontos anatômicos.
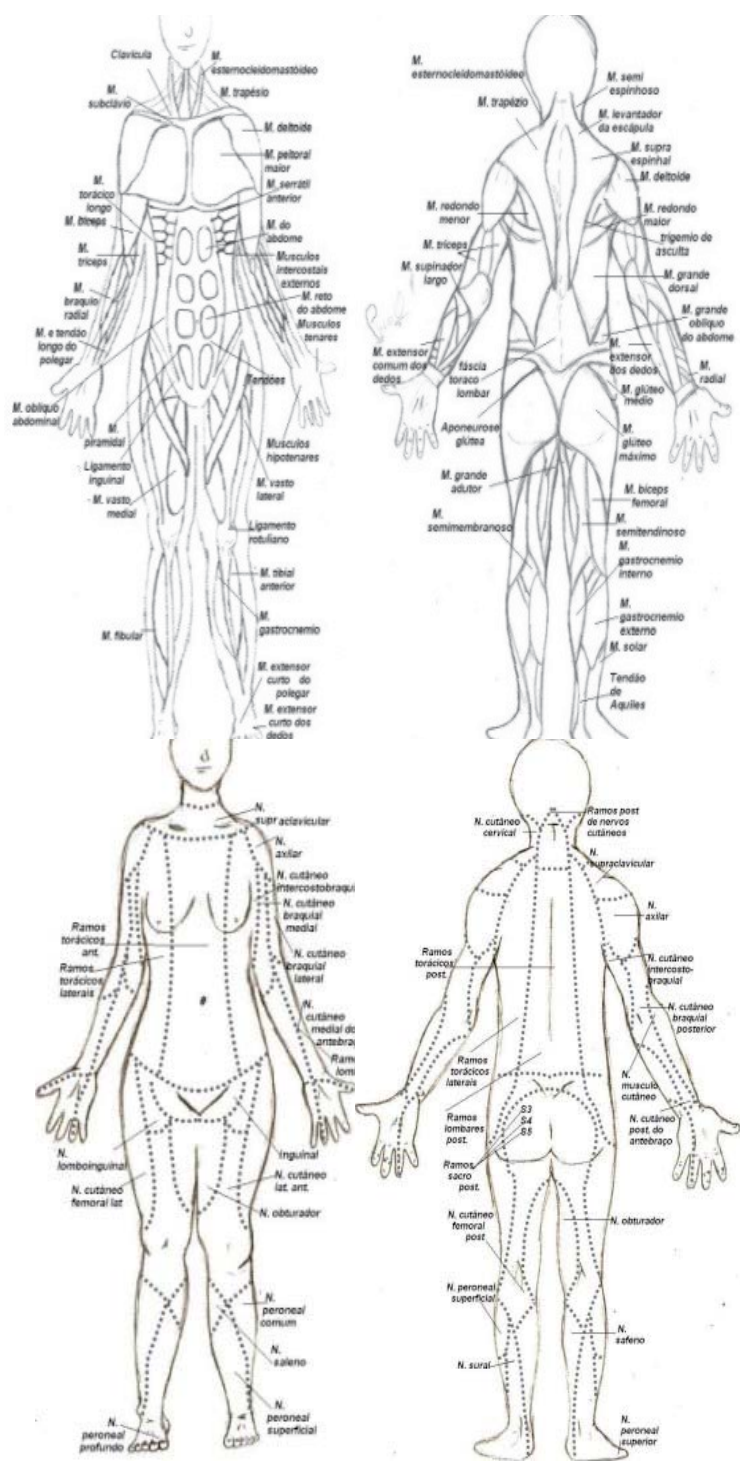

\PAJMT

FIGURA 2 Corpo humano demonstrando músculos e nervos, anteriores e posteriores.

Nas figuras 3 e 4 é possível verificar imagem da região dos glúteos e cóccix. Percebe-se com clareza, que a região glútea direita apresenta ponto com significativa diferença térmica, se comparado com as regiões circunvizinhas, apresentando diferença de temperatura de $2,1^{\circ} \mathrm{C}$. A região glútea esquerda também apresenta ponto com diferença significativa de temperatura quando comparado com a região circunvizinha, de $1,6^{\circ} \mathrm{C}$. A região coccígea, também verificada nesta imagem, apresenta uma diferença de temperatura, entre o ponto quente e áreas ao redor, de $1,7^{\circ} \mathrm{C}$. 


\section{PAN AMERICAN JOURNAL OF MEDICAL THERMOLOGY}

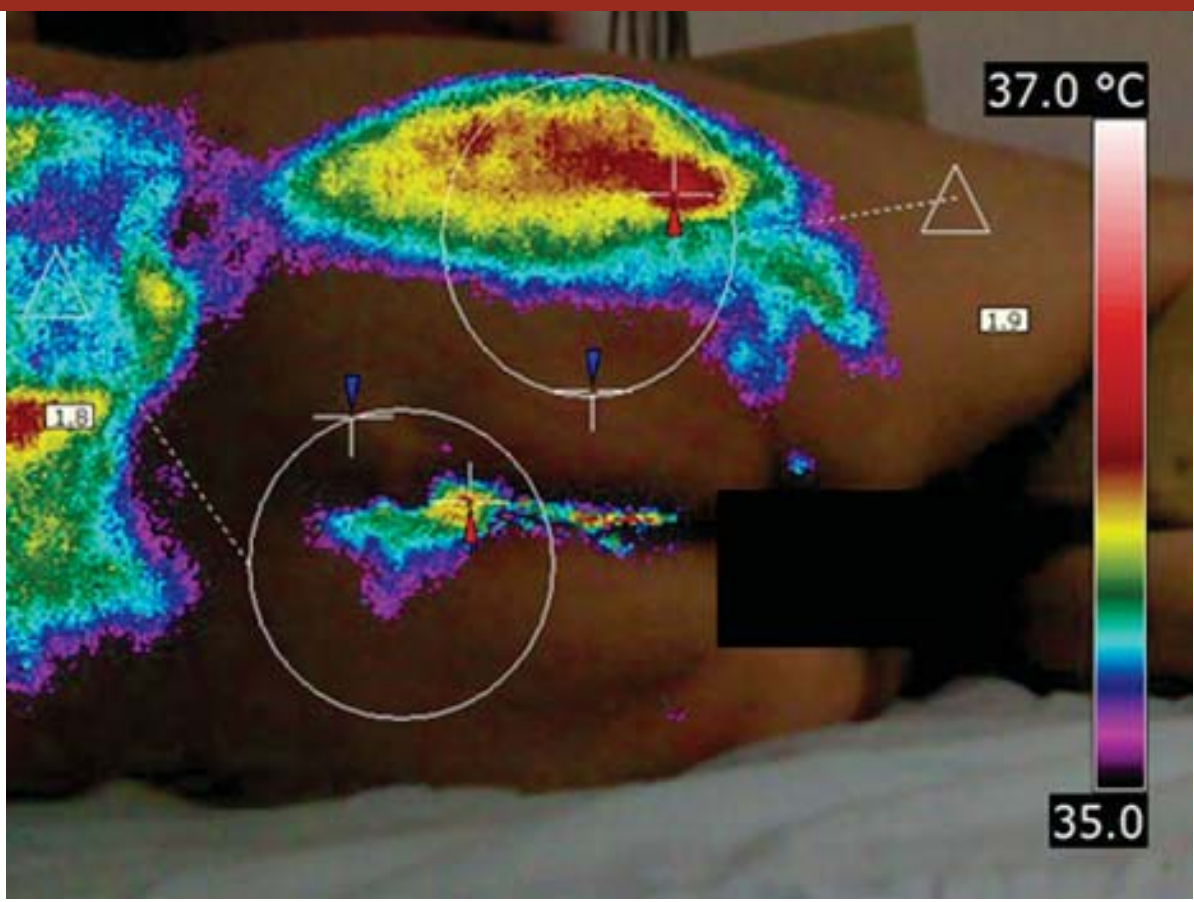

FIGURA 3 Regiões de glúteos direito $\left(\Delta 1,9^{\circ} \mathrm{C}\right)$ e coccígea $\left(\Delta 1,8^{\circ} \mathrm{C}\right)$, comparação com hotspot e regiões circunvizinhas

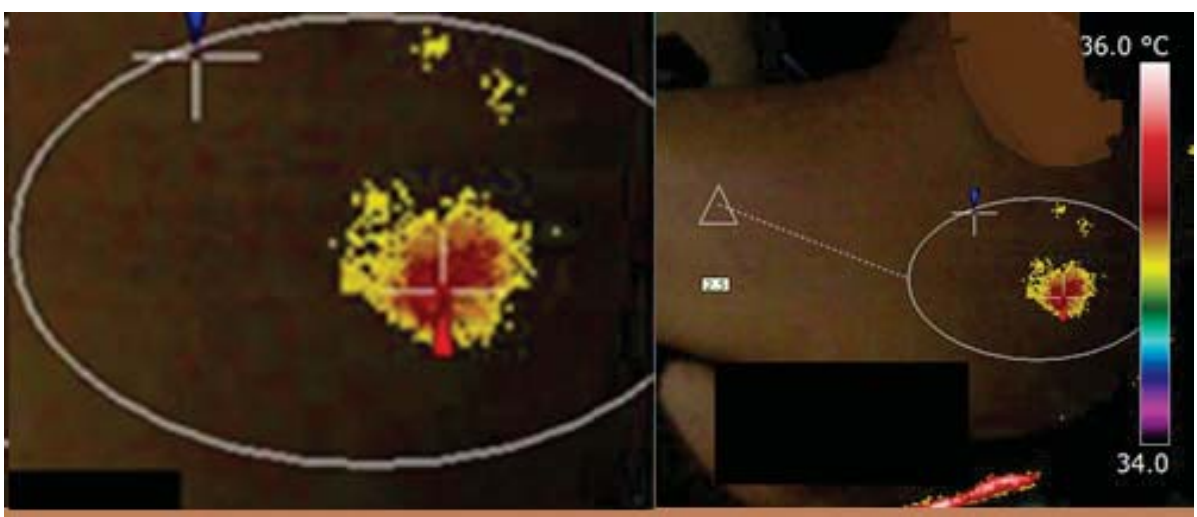

FIGURA 4 Área glútea esquerda $\left(\Delta 2,5^{\circ} \mathrm{C}\right)$, hotspot comparado com regiões circunvizinhas

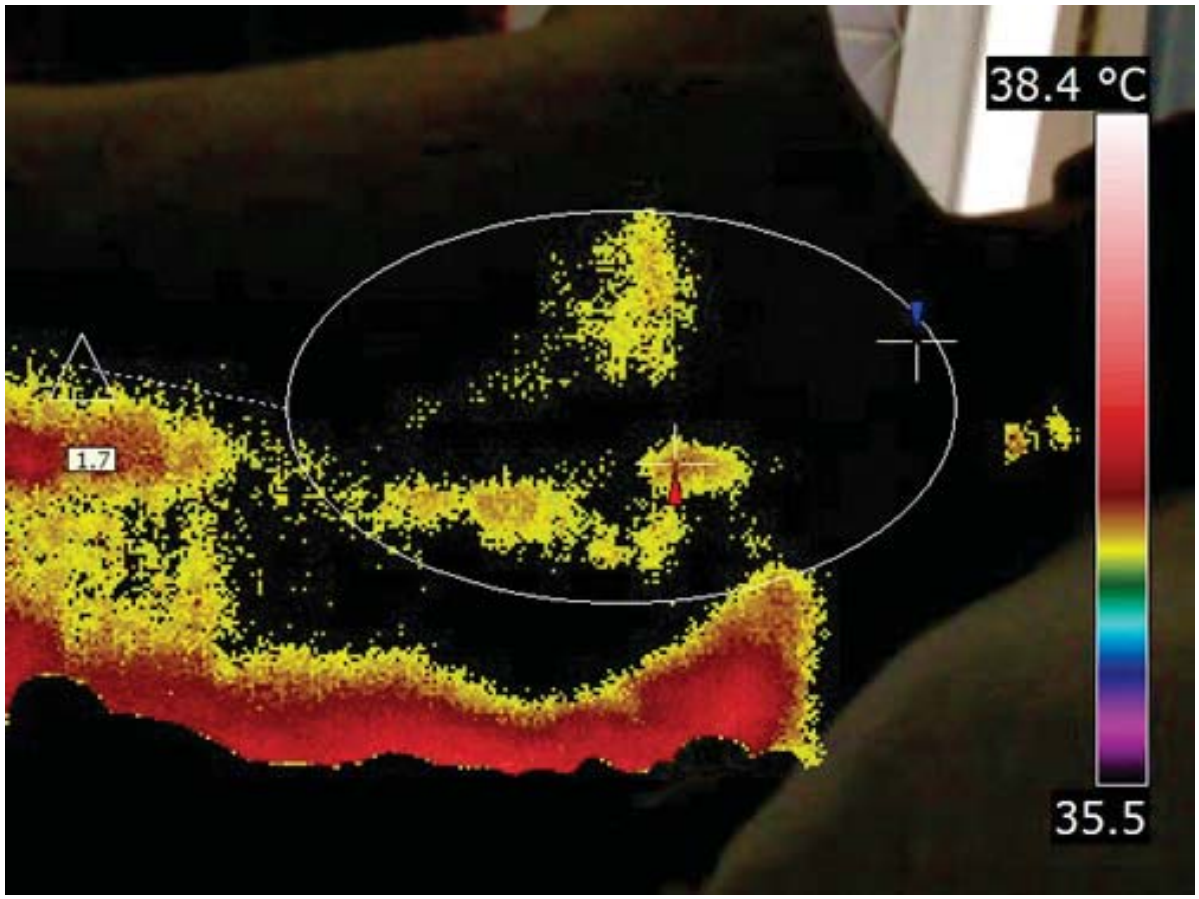

FIGURA 5 Análise de temperaturas da região de escápulas, $\Delta 1,7^{\circ} \mathrm{C}$ (pontos quentes e mais frios) indicando pontos de pressão 
Outro ponto analisado pelo exame de Termografia é a região escapular, onde também se localizam pontos quentes. $\mathrm{Na}$

figura 5, dentro da circunferência, o ponto mais quente encontra-se onde está o marcador vermelho e o mais frio onde está o marcador azul.

\section{DISCUSSÃo}

Os pontos de desconforto descritos na figura 1 são demonstrados nos termogramas pelas diferenças de temperatura entre a região do incômodo e região sem dor. As regiões de glúteos demonstram diferenças de temperatura de $\triangle 1,9^{\circ} \mathrm{C}$ e coccígea de $\triangle 1,8^{\circ} \mathrm{C}$, ao mesmo tempo que a região de glúteo esquerdo tem a diferença de $2,5^{\circ} \mathrm{C}$ em comparação com regiões circunvizinhas, o que demonstra considerável diferença de temperatura. Do mesmo modo a região escapular, apresenta uma diferença de temperatura significativa de $1.7^{\circ} \mathrm{C}$

Judy ${ }^{10}$ realizou um estudo com a Termografia para prever o desenvolvimento de úlcera de pressão, e como método, foram avaliados 100 pacientes, durante um ano e meio, com risco de desenvolver escaras. Foi utilizado, para comparação, a Escala de Braden (instrumento de avaliação do risco para desenvolvimento de úlceras por pressão). No experimento, a câmera infravermelha focou nas proeminências ósseas, como sacro e escápulas, por exemplo. ${ }^{9}$ Esses estudos apontaram que a Termografia classificou corretamente cinco localizações anatômicas de alto risco, que desenvolvem uma úlcera de pressão, usando a diferença de temperatura no alvo, área circulada para cálculo de temperatura, diferença $(\Delta)$ de no mínimo $1,5^{\circ} \mathrm{C}$ entre as regiões com lesão e partes vizinhas saudáveis. E, embora as técnicas de avaliação de risco nunca devem substituir o julgamento clínico, a Termografia pode fornecer informações valiosas sobre pontos específicos no risco de úlceras de pressão. $^{10}$

Esse estudo demonstrou que, por meio de exame Termográfico, pode-se auxiliar a monitoração de pacientes acamados que não se movimentam e que podem sofrer com ulceras de pressão, pelo atrito de seu corpo com a cama, cadeiras, etc.

\section{AGRADECIMENTOS}

Ao Professor Dr. Marcos Leal Brioschi InfraRedMed - HCFMUSP.

\section{REFERÊNCIAS}

1. Paiva, Lucila Corsino de. Úlcera de pressão em pacientes internados em um hospital universitário em Natal/RN: condições predisponentes e fatores de risco. Lucila Corsino de Paiva. - Natal, 2008. 99.

2. Hoffjan, S., Akkad, D.A. The genetics of multiple sclerosis: an update 2010. Molecular and Cellular Probes, p. 237-243, 2010.

3. Bienes, Gabriel; Oliveira, Enedina Maria Lobato; Bichuetti, Denis Bernardi. Esclerose múltipla. Multiple Sclerosis. Rev Bras Med, v. 71, n. 12, p. 37-45, 2014.

4. Oliveira, E. M. L.; Annes, M.; Oliveira A. S. B.; Gabbai, A. A. Esclerose múltipla. Estudo clínico de 50 pacientes acompanhados no ambulatório de neurologia UNIFESPEPM. Arq. Neuropsiquiatria. vol.57 n.1 São Paulo Mar. 1999.

5. Ferreira, M. L. B.; Machado, M. Í. M.; Vilela, M. L.; Guedes, M. J.; Ataíde, L. JR; Santos, S.; Laurentino, S. G. Epidemiologia de 118 Casos de Esclerose Múltipla com Seguimento de 15 anos no Centro de Referência do Hospital da Restauração de Pernambuco. Arq Neuropsiquiatria, 62(4):1027-1032, 2004.

6. Almeida, L. H. R. B., de Oliveira, F. T. M., da Silva, M. K. M., Rocha, F. C., do Nascimento, F. C. L., \& DA SILVA, G. R. F. Conhecimento dos profissionais de saúde sobre esclerose múltipla. Acta Scientiarum. Health Science, 33(2), 133-138, 2011.

7. Altoé, L, Oliveira Filho, D. Termografia infravermelha aplicada à inspeção de edifícios. Acta Tecnológica; 7 : 5559, 2012.

8. Reisemberger, G.G. Analise e triagem de tumor benigno a partir de imagens de infravermelho. Dissertação (Mestrado em Engenharia Mecânica) - Setor de Tecnologia, Universidade Federal do Paraná, Curitiba, 2014.

9. Bronzino, J. D. The biomedical engineering handbook: 

Pajma Medical Devices and systems, 3a edição. New York: CRC
Press, 2006.

10. Judy, D., Brooks, B., FennieK, Lyder, C., Burton, C.

Improving the detection of pressure ulcers using the TMI

ImageMed system. Adv Skin Wound Care; 24(1):18-24,

2011. 